\title{
AN EXAMPLE OF FOURIER-MUKAI PARTNERS OF MINIMAL ELLIPTIC SURFACES
}

\author{
HOKUTO UEHARA
}

\begin{abstract}
Let $X$ and $Y$ be smooth projective varieties over $\mathbb{C}$. We say that $X$ and $Y$ are D-equivalent (or, $X$ is a Fourier-Mukai partner of $Y$ ) if their derived categories of bounded complexes of coherent sheaves are equivalent as triangulated categories. The aim of this short note is to find an example of mutually D-equivalent but not isomorphic relatively minimal elliptic surfaces.
\end{abstract}

\section{Introduction}

Let $X$ be a smooth projective variety over $\mathbb{C}$. The derived category $D(X)$ of $X$ is a triangulated category whose objects are bounded complexes of coherent sheaves on $X$. A Fourier-Mukai (FM) transform relating smooth projective varieties $X$ and $Y$ is an equivalence of triangulated categories $\Phi: D(X) \rightarrow D(Y)$. If there exists an FM transform relating $X$ and $Y$, we call $X$ an FM partner of $Y$. We also say that $X$ and $Y$ are D-equivalent. Moreover we say that $X$ and $Y$ are K-equivalent if there exist a smooth projective variety $Z$ and birational morphisms $f: Z \rightarrow X, g: Z \rightarrow Y$ such that $f^{*} K_{X} \sim g^{*} K_{Y}$. It is conjectured by Kawamata (Conjecture 1.2 in [7]) that given birationally equivalent smooth projective varieties $X$ and $Y$, they are D-equivalent if and only if they are Kequivalent. In this note, we construct a counterexample to his conjecture. More precisely, we have:

Main Theorem . (i) Let $p$ be a positive integer. Then there is a rational elliptic surface $S(p)$ such that $S(p)$ has a singular fiber of type ${ }_{p} I_{0}$ and at least three non-multiple singular fibers of different Kodaira's types.

(ii) Let $N$ be a positive integer and $p$ a prime number such that $p>6(N-1)+1$. Then there are rational elliptic surfaces $T_{i},(1 \leq i \leq N)$ such that $T_{i} \neq T_{j}$ for $i \neq j$ and every $T_{i}$ is an FM partner of $S(p)$. As a special case, $S=S(11)$ has an $F M$ partner $T$ such that $T \not S$. These $S$ and $T$ are birational, D-equivalent but not K-equivalent.

Note that if $X$ and $Y$ are K-equivalent, they are isomorphic in codimension 1 (Lemma 4.2, [7]). In particular, if surfaces $S$ and $T$ are not isomorphic, they are not K-equivalent. Hence, in (ii), the statement for $p=11$ follows from the one for arbitrary $p$.

Received December 1, 2003.

2000 Mathematics Subject Classification. Primary: 14J27, 14E26. 
Before ending Introduction, we give a few remarks to Main Theorem. For a smooth projective variety $X$, it is an interesting problem to find the set of isomorphic classes of FM partners of $X$. In connection with this problem, we have the following.

Theorem 1.1 (Theorem 1.1, [2] and Theorem 1.6, [7]). Assume that $X$ and $Y$ are D-equivalent smooth projective surfaces but not isomorphic to each other. Then we know that one of the following holds.

(i) $X$ and $Y$ are K3 surfaces.

(ii) $X$ and $Y$ are abelian surfaces.

(iii) $X$ and $Y$ are elliptic surfaces with the non-zero Kodaira dimension $\kappa(X)=$ $\kappa(Y)$.

Using Theorem 1.1, we obtain the complete answer to the problem mentioned above in dimension 2 ([2], see also [7]). It is well-known that the cases (i) and (ii) in Theorem 1.1 really occur. More strongly, we have:

Theorem 1.2 ([8] and [6]). Let $N$ be a positive integer. Then there are K3 (respectively, abelian) surfaces $T_{i},(1 \leq i \leq N)$ such that $T_{i} \neq T_{j}$ for $i \neq j$ and all $T_{i}$ 's are D-equivalent each other.

Our Main Theorem means that the case (iii) in Theorem 1.1 really occurs, and a similar result to Theorem 1.2 is true for elliptic surfaces.

In contrast to Main Theorem and Theorem 1.2, it is predicted that given a smooth projective variety $X$, the set of isomorphic classes of FM partners of $X$ is finite. Actually this is known for the 2-dimensional case ([2] and [7]).

Notation and conventions. All varieties are defined over $\mathbb{C}$ and "elliptic surface" always means "relatively minimal elliptic surface" in this note. For a set $I$, we denote by $|I|$ the cardinality of $I$.

\section{The proof of Main Theorem}

We need some standard notation and results before giving the proof. Let $\pi: S \rightarrow C$ be an elliptic surface. For an object $E$ of $D(S)$, we define the fiber degree of $E$

$$
d(E)=c_{1}(E) \cdot f
$$

where $f$ is a general fiber of $\pi$. Let us denote by $\lambda_{S / C}$ the highest common factor of the fiber degrees of objects of $D(S)$. Equivalently, $\lambda_{S / C}$ is the smallest number $d$ such that there is a holomorphic $d$-section of $\pi$. For integers $a>0$ and $i$ with $i$ coprime to $a \lambda_{S / C}$, by [1] there exists a smooth, 2-dimensional component $J_{S}(a, i)$ of the moduli space of pure dimension one stable sheaves on $S$, the general point of which represents a rank $a$, degree $i$ stable vector bundle supported on a smooth fiber of $\pi$. There is a natural morphism $J_{S}(a, i) \rightarrow C$, taking a point representing a sheaf supported on the fiber $\pi^{-1}(x)$ of $S$ to the point $x$. This morphism is a minimal elliptic fibration $([1])$. Put $J^{i}(S):=J_{S}(1, i)$. Obviously, $J^{0}(S) \cong J(S)$, the Jacobian surface associated to $S$, and $J^{1}(S) \cong S$. 
Fix an elliptic surface with a section $\pi: B \rightarrow C$. Let $\eta=$ Spec $k$ be the generic point of $C$, where $k=k(C)$ is the function field of $C$, and let $\bar{k}$ be the algebraic closure of $k$. Put $\bar{\eta}=\operatorname{Spec} \bar{k}$. We define the Weil-Chatelet group $W C(B)$ by the Galois cohomology $H^{1}\left(G, B_{\eta}(\bar{k})\right)$. Here $G=\operatorname{Gal}(\bar{k} / k)$ and $B_{\eta}(\bar{k})$ is the group of points of the elliptic curve $B_{\eta}$ defined over $\bar{k}$. Suppose that we are given a pair $(S, \varphi)$, where $S$ is an elliptic surface $S \rightarrow C$ and $\varphi$ is an isomorphism $J(S) \rightarrow B$ over $C$, fixing their 0 -sections. Then we have a morphism

$$
B_{\eta} \times S_{\eta} \rightarrow J(S)_{\eta} \times S_{\eta} \rightarrow S_{\eta} .
$$

Here the first morphism is induced by $\varphi^{-1} \times i d_{S}$ and the second is given by translation. We obtain a principal homogeneous space $S_{\eta}$ of $B_{\eta}$. Since this correspondence is invertible and the group $H^{1}\left(G, B_{\eta}(\bar{k})\right)$ classifies isomorphic classes of principal homogeneous spaces of $B_{\eta}$, we know that $W C(B)$ consists of all isomorphic classes of pairs $(S, \varphi)$. Here two pairs $(S, \varphi)$ and $\left(S^{\prime}, \varphi^{\prime}\right)$ are isomorphic if there is an isomorphism $\alpha: S \rightarrow S^{\prime}$ over $C$, such that $\varphi^{\prime} \circ \alpha_{*}=\varphi$, where $\alpha_{*}: J(S) \rightarrow J\left(S^{\prime}\right)$ is the isomorphism induced by $\alpha$ (fixing 0 -sections).

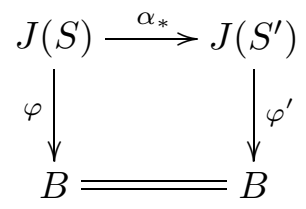

There is a short exact sequence (page 185, [4] or page $38,[3]$ )

$$
0 \rightarrow \operatorname{Sh}(B) \rightarrow W C(B) \rightarrow \bigoplus_{t \in C} H_{1}\left(B_{t}, \mathbb{Q} / \mathbb{Z}\right) \rightarrow 0,
$$

if $B$ is not the product $C \times E$, where $E$ is an elliptic curve. The group $\operatorname{Sh}(B)$ is called Tate-Shafarevich group and it is the subgroup of $W C(B)$ which consists of all isomorphic classes of pairs $(S, \varphi)$ such that $S$ does not have multiple fibers. For a rational surface $B$, it is known that $\operatorname{Sh}(B)$ is trivial (Example 1.5.12, [5]).

Now we are in position to prove Main Theorem.

Proof. (i) By the Persson's list [9], there is a rational elliptic surface $B \rightarrow C$ having a section and three singular fibers of type $I I I^{*}, I_{2}, I_{1}$ (there are many other choices for $B)$. Fix a point $t_{0} \in C$ such that $B_{t_{0}}$ is smooth. Take an element $\xi=\left(\xi_{t}\right)$ of $W C(B) \cong \bigoplus_{t \in C} H_{1}\left(B_{t}, \mathbb{Q} / \mathbb{Z}\right)$ such that $\xi_{t_{0}}$ is of order $p$ and $\xi_{t}=0$ for other $t$. Then the surface $\pi: S(p) \rightarrow C$ corresponding to $\xi$ is an elliptic surface with desired singular fibers. We can check that $S(p)$ is rational, for instance, by Proposition 1.3.23, [5].

(ii) Put $S=S(p)$. Because every $(-1)$-curve on $S$ is a $p$-section of $\pi$, we know that $\lambda_{S / C}=p$. For $i \in \mathbb{Z}$, there is an isomorphism $\varphi_{i}: J\left(J^{i}(S)\right) \rightarrow B$ such that $\left(J^{i}(S), \varphi_{i}\right)$ corresponds to $i \xi \in W C(B)$ ([3], page 38). By Theorem 2.2 , each $J^{i}(S)$ is mutually D-equivalent for $1 \leq i<p$. We can also conclude that $J^{i}(S)$ is rational, since $\kappa\left(J^{i}(S)\right)=-\infty$ and the Euler numbers $e\left(J^{i}(S)\right)$ 
and $e(S)$ coincide by Proposition 2.3, [2] (we can check the rationality also by using Proposition 1.3.23, [5]). Put

$$
I=\{1, \ldots, p-1\}, \quad I(a)=\left\{i \in I \mid J^{i}(S) \cong J^{a}(S)\right\}
$$

for $a \in I$. Then there are $i_{1}, \ldots, i_{M} \in I$ such that $I=\coprod_{k=1}^{M} I\left(i_{k}\right)$ (disjoint union).

Claim 2.1. For all $a \in I,|I(a)| \leq 6$.

If Claim 2.1 is true, we have $6 M \geq|I|=p-1$. By the assumption $p>$ $6(N-1)+1$, we have $M \geq N$, which completes the proof of Main Theorem.

Let us start the proof of Claim 2.1.

Step 1. For each $i \in I(a)$, we fix an isomorphism $\alpha_{i}: J^{a}(S) \rightarrow J^{i}(S)$. Because the rational surface $J^{a}(S)$ has a unique elliptic fibration, there exists $\delta \in$ Aut $C$ such that the following diagram is commutative.

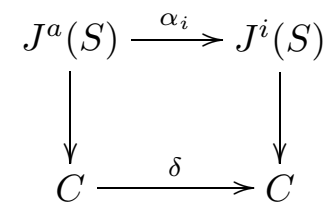

This makes the following diagram commutative.

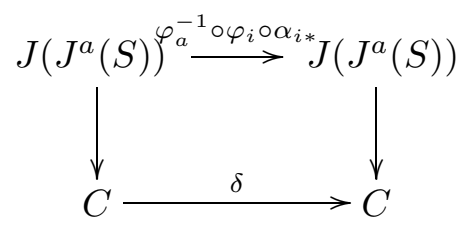

By our assumption, $J\left(J^{a}(S)\right)$ has at least three singular fibers of different Kodaira's types. Hence $\delta$ must be the identity on $C \cong \mathbb{P}^{1}$ and then we can say that every $\alpha_{i}$ is an isomorphism over $C$.

Step 2. By Step 1, we know that $\varphi_{i} \circ \alpha_{i *} \circ \varphi_{a}^{-1}$ is an automorphism of $B$ over $C$, fixing the 0 -section. Put $\gamma_{i}=\varphi_{i} \circ \alpha_{i *} \circ \varphi_{a}^{-1}$.

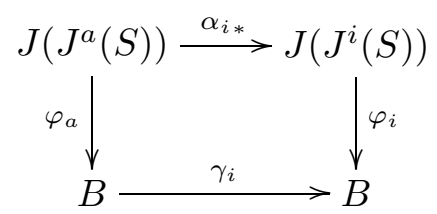

Suppose $\gamma_{i}=\gamma_{j}$ for $i, j \in I(a)$, then by the isomorphism $\alpha_{j} \circ \alpha_{i}^{-1}$, we see that $\left(J^{i}(S), \varphi_{i}\right)$ is isomorphic to $\left(J^{j}(S), \varphi_{j}\right)$ and hence $i \xi=j \xi$ in $W C(B)$. Because the order of $\xi$ is $p$, we obtain $i=j$. Since the order of the group of automorphism of $B$ over $C$ fixing the 0 -section is at most 6 , we get $|I(a)| \leq 6$. This finishes the proof.

Theorem 2.2 (Proposition 4.4, [2]). Let $\pi: S \rightarrow C$ be an elliptic surface and $T$ a smooth projective variety. Assume that the Kodaira dimension $\kappa(S)$ is nonzero. Then the following are equivalent. 
(i) $T$ is an FM partner of $S$.

(ii) $T$ is isomorphic to $J^{b}(S)$ for some integer $b$ with $\left(b, \lambda_{S / C}\right)=1$.

I would like to express my hearty thanks to Professors Akira Ishii, Noboru Nakayama and Takeshi Kajiwara for useful conversation. I am very grateful to the referee for his/her invaluable suggestions.

\section{References}

[1] T. Bridgeland, Fourier-Mukai transforms for elliptic surfaces, J. Reine Angew. Math. 498 (1998), 115-133.

[2] T. Bridgeland, A. Maciocia, Complex surfaces with equivalent derived categories, Math. Z. 236 (2001), 677-697.

[3] R. Friedman, Vector bundles and SO(3)-invariants for elliptic surfaces, J. Amer. Math. Soc. 8 (1995), 29-139.

[4] - Algebraic surfaces and holomorphic vector bundles. Universitext. SpringerVerlag, New York, 1998.

[5] R. Friedman, J. Morgan, Smooth four-manifolds and complex surfaces. Ergebnisse der Mathematik und ihrer Grenzgebiete (3), 27. Springer-Verlag, Berlin, 1994. x+520 pp

[6] S. Hosono, B. H. Lian, K. Oguiso, S.-T. Yau. Kummer structures on a K3 surfaceAn old question of T. Shioda, math.AG/0202082

[7] Y. Kawamata, D-equivalence and K-equivalence, J. Differential Geom. 61 (2002), 147171.

[8] K. Oguiso, K3 surfaces via almost-primes, Math. Res. Lett. 9 (2002), 47-63.

[9] U. Persson, Configurations of Kodaira fibers on rational elliptic surfaces. Math. Z. 205 (1990), 1-47.

Department of Mathematics, Kyoto University, Kyoto, 606-8502, Japan

E-mail address: hokuto@kurims.kyoto-u.ac.jp 